\title{
Reciprocal Space Signals Arising from Interference of Core-Loss Electrons in the EMCD Geometry
}

\author{
K. Leifer ${ }^{*}$, S. Rubino ${ }^{*}$, J. Rusz $^{* *}$, H. Lidbaum ${ }^{*}$, B. Hjörvarsson ${ }^{* * *}$, P. Oppeneer ${ }^{* *}$, O. Eriksson ${ }^{* *}$ \\ * Inst. Electron Microscopy and Nano-Engineering, Department of Engineering, Uppsala University, \\ 75221 Uppsala, Sweden \\ ** Inst. Theoretical Physics, Department of Physics and Astronomy, Uppsala University, 75221 \\ Uppsala, Sweden
}

Inst. of Materials Physics, Department of Physics and Astronomy, Uppsala University, 75221 Uppsala, Sweden

The discovery of magnetic circular dichroism [1] enabled for the first time the determination magnetic orbital/spin moment ratios in the energy filtered diffraction patterns [2]. This magnetic moment ratio is a key parameter for the understanding nanomagnetism phenomena magnetic materials. Furthermore, the acquisition of such EMCD signals either with a focused electron probe or in energy filtered image series demonstrate a resolution of the EMCD technique in the few nanometer range [3]. All of these approaches to obtain the EMCD signal have in common that the EMCD signal is inherently weak, i.e. must be optimised and that the detailed distribution of the signal in reciprocal space must be known in order to obtain and certainly to interpret the intensities. Here, we show which procedures can be applied to optimize the EMCD signal experimentally and propose data treatment procedures. Both, acquisition optimization and data treatment are guided by simulations of the EMCD signal. The optimization of the Signal/noise ratio has lead to the first real space map of the EMCD signal. We equally show that the influence of deconvolution on the EMCD signal must be considered in order to obtain a quantitative determination of the orbital/spin magnetic moment (figure 1).

The importance of simulations in guiding EMCD experiments leads us to the exploration of new signals appearing in energy filtered reciprocal space patterns. We developed an extended formalism of current sum-rules leading to the creation of a number of physical property operators, where the spin and orbital operators are a subset. These operators are shown to have different symmetries of their signal in reciprocal space. Thus, energy filtered diffraction patterns can be decomposed into different operators. A further refinement of the simulations shows that a fine structure appears in the EMCD signal itself. This fine structure scales with the sample thickness (figure 2). The EMCD technique is thus ready to address questions in modern nanomagnetism in both real and reciprocal space.

\section{References}

[1] P. Schattschneider et al., Nature 441 (2006) 486.

[2] H. Lidbaum, J. Rusz, A. Liebig, B. Hjörvarsson, P. Oppeneer, O. Eriksson, E. Coronel, K. Leifer, Phys. Rev. Lett 101 (2009) 037201.

[3] P. Schattschneider et al., Phys. Rev. Lett. 78 (2008) 104413, Lidbaum et al., Ultramicr. 110 (2010) 1380, Stöger-Pollach et al., Micron, in press 2011.

[4] The authors would like to acknowledge support of this work from the Swedish Science Council, the Swedish Foundation for International Cooperation in Research and Higher education STINT and the KAW Foundation 


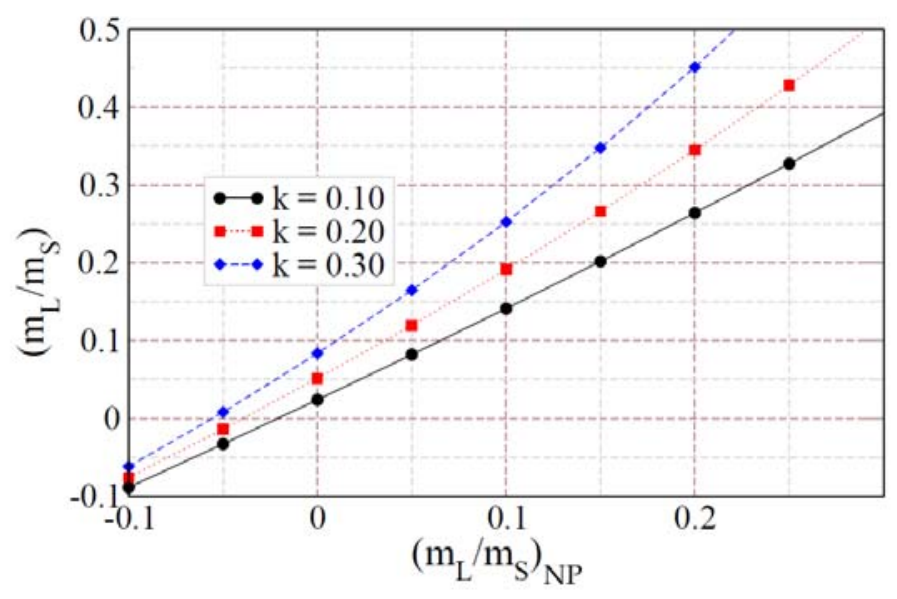

Fig. 1 Influence of plural scattering on the orbital/spin magnetic moment ratio $\mathrm{m}_{\mathrm{L}} / \mathrm{m}_{\mathrm{S}}$. The $\left(\mathrm{m}_{\mathrm{L}} / \mathrm{m}_{\mathrm{S}}\right)_{\mathrm{NP}}$ : ratio taken from spectra that have not undergone plural scattering; $\left(\mathrm{m}_{\mathrm{L}} / \mathrm{m}_{\mathrm{S}}\right)$ : same spectra that have undergone plural scattering. The factor $\mathrm{k}$ describes the strength of plural scattering. It can clearly be observed that even when $\mathrm{k}$ is small, there is a significant discrepancy between the $\left(\mathrm{m}_{\mathrm{L}} / \mathrm{m}_{\mathrm{S}}\right)$ and $\left(\mathrm{m}_{\mathrm{L}} / \mathrm{m}_{\mathrm{S}}\right)_{\mathrm{NP}}$.

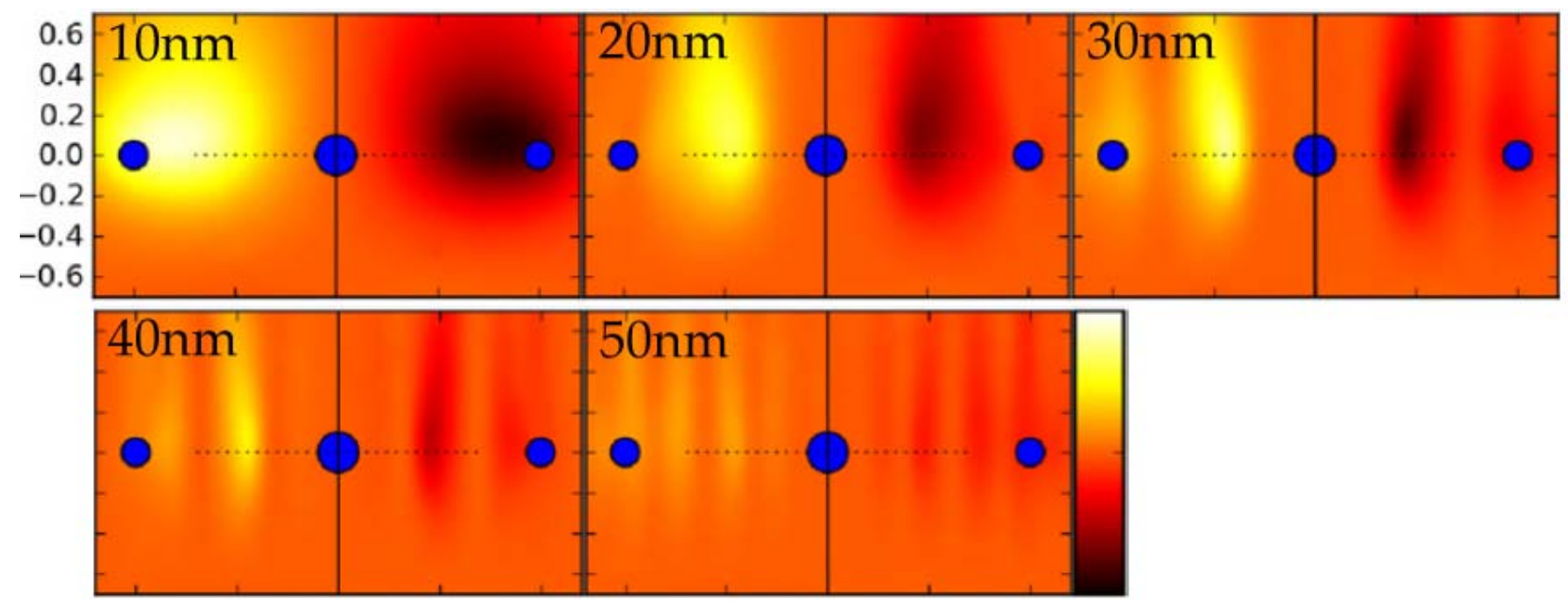

Fig. 2 Simulation of dichroic signal in reciprocal space and in 3-beam-case orientation as a function of thickness. The maps are shown at an energy loss of $708 \mathrm{eV}$ and the blue dots represent the positions of (-200), (200) reflections and the 0-beam. The EMCD signal depends strongly on the thickness of the sample and a fringe like pattern appears at sample thicknesses above $30 \mathrm{~nm}$. 